\title{
Diagnóstico para un modelo de análisis autopoiético hacia proyectos de diseño
}

Azael Pérez Peláez 1

Miguel Ángel Rubio Toledo²

Universidad Autónoma del Estado de México. México

RECIBIDO: 28.09.2016 / ACEPTADO: 27.02.2017

\section{Resumen}

Se pretende caracterizar el concepto de autopoiésis para definir al sistema vivo para encontrar la esencia que puede ser reproducida en proyectos de diseño. El comprender a lo vivo como un sistema autopoiético permite puntualizar las características esenciales que lo definen como tal para llevar a cabo un proceso biomimético que reproduzca dichas características en un modelo metodológico aplicable a procesos de creación antrópica.

Palabras clave: modelo, autopoiésis, proyectos, diseño, biomímesis.

\section{Diagnosis for a autopoietic model analysis towards design projects}

\begin{abstract}
This paper aims to characterize the concept of autopoiesis to define the living system to find the essence that can be reproduced on design projects. Understanding the life as an autopoietic system allows to point out the essential characteristics that define it as such to carry out a biomimetic process to reproduce these features in an applicable methodological model applicable in anthropic process of creation.
\end{abstract}

Keywords: model, autopoiesis, projects, design, biomimesis.

\footnotetext{
${ }^{1}$ Azael Pérez Peláez es Licenciado en Diseño Gráfico, Maestría en Diseño con mención honorífica. Artista ambiental y desarrollador de estrategias, cursos y talleres de enseñanza artística y ambiental. Con exposiciones artísticas en México y España. Realizó una estancia de investigación sobre Diseño Relacional aplicado al arte en la Universidad Politécnica de Valencia. Actualmente investiga el campo del diseño biomimético y sus fundamentos en el programa de Doctorado en Diseño de la Facultad de Arquitectura y Diseño de la Universidad Autónoma del Estado de México. E-mail: freudspid@hotmail.com

2 Miguel Ángel Rubio Toledo es Licenciado en Diseño Gráfico, MBA en Artes Visuales y Doctor en Ciencias Sociales. Profesor investigador de tiempo completo del Centro de Investigación en Arquitectura y Diseño de la Universidad Autónoma del Estado de México. Ha escrito diversos documentos indexados, capítulos de libros y libros, y participado en diversas conferencias en México y en el extranjero, principalmente relacionados con la producción y el consumo simbólico, metodología, ética y responsabilidad social en el diseño, y sobre el Diseño estratégico. E-mail: miguelblond72@yahoo.com.mx
} 


\section{Qué es autopoiésis}

En 1975, Humberto Maturana se dio a la tarea de encontrar la particularidad definidora de lo vivo, responder a la cuestión de ¿qué es lo que hace que un ser vivo sea vivo? Su objetivo era saber el punto exacto en el que un sistema viviente podía ser definido como tal encontrando el sistema organizacional esencial (Maturana, 1998).

Cuando Maturana propone al concepto de autopoiésis, como patrón esencial de organización de los sistemas vivientes, desató una revolución de modelos que transportaron este modelo hacia lis sostemas sociales (Luhman, 1997). Aun cuando Maturana advierte del riesgo de confundir al sistema viviente con el sistema social al complejizarse la intersección de las estructuras que los componen, dichos modelos prosperaron en con una serie de sobrevalores que poco a poco han diluido las características constantes del modelo inicial.

\section{Cómo construir un modelo de análisis autopoiético}

Para poder construir un modelo de análisis autopoiético que sirva para estudiar el comportamiento organizacional de los diseños humanos, es necesario regresar al origen del modelo autopoiético y esquematizar de forma puntual el patrón de antidispersión cíclica al que se refiere Maturana cuando postula a la autopoiésis como definidor esencial de lo vivo.

Aprovechando esta definición se puede reconocer que ciertos sobrevalores agregados a la idealización de los sistemas antrópicos pueden ser clasificados en 2 grupos que ayuden a distinguir lo definidor de lo dispersor.

Se llamará al primer grupo características dispersoras para referirse a aquellas variables idealizadas que pueden presentarse a la hora del planteamiento y desarrollo del diseño y que por su naturaleza no esencial se recomienda prescindir del método de abordaje para lograr una potenciación de las ventajas del diseño biomimético:

Características dispersoras:

- Hibridación

- Complementariedad

- Autoregulación

- Migración-desplazamiento

- Evitación

- Mitigación

- Reintegración

- Autocreación

- Inmortalidad

- Retroalimentación

- Espontaneidad 
Estas características se pueden sumar a muchas otras que de forma superflua pueden llegar a obstaculizar el proceso de diseño en busca de valores agregados. Esta tendencia se puede ver acentuada cuando en correspondencia a perspectivas hibridadas de métodos de diseño se busca responder a factores de mejoras, certificaciones, aceptación social, moda, popularidad o tendencia.

Para mencionar un ejemplo se puede hablar de las característica agregadas que se sumarían si se combina el procesoo de biomímesis autopoiética con el de la sustentabilidad clásica. Las esferas de partida de los métodos de abordaje en la sustentabilidad requieren de contemplaciones sociales, ambientales y económicas que sumarían características tales como; Reusabilidad o reciclablidad, retroalimentación integral de los elementos componentes, contemplación del ciclo de vida del producto, modularidad obligada o correspondencia formal, aceptación e integración social, etc.

Todos estos factores de sobrevalor son loables en su particularidad específica, es decir, pueden ser contemplados en el proceso de diseño, siempre y cuando no se olvide que la esencia del designio detonante, sobre todo en el caso del diseño biomimético, recaiga sobre el patrón de antidispersión del objeto a diseñar, es decir en la esencia de lo vivo transportado a la creación humana.

Se nombrará al segundo grupo características definidoras en prosecución del objetivo de encontrar el mínimo común definidor de lo vivo y sus particularidades esenciales. Maturana describe a la organización autopoiética como un ciclo de auto detonación que se comporta de forma que evita su propia dispersión y que al mismo tiempo conserva su carácter de movimiento entrópico. En esta descripción se describe un patrón de antidispersión cíclica, que a su vez se compone de tres fuerzas detonadoras y cuatro comportamientos esenciales.

Características definidoras:

- Fuerzas detonadoras

-Entropía

-Muerte

-Desarrollo

-Comportamientos

- Tendencia de dispersión

- Tendencia de conservación

-Autoregulación

-Actividad relacional

\section{Propuesta de Modelo}

Este patrón de antidispersión cíclica se ha caracterizado entonces en 7 componentes esenciales; 4 comportamientos que caracterizan a los sistemas vivos, y 3 fuerzas esenciales que combinadas detonan la auto-organización a la que hace referencia el concepto de autopoiésis. 
Este patrón puede explicarse mediante un modelo esquematizado de forma que se comprendan los movimientos y dinámicas que relacionan a las características y fuerzas entre ellas.

En la figura 1 se puede ver un esquema donde se describe a la fuerza detonadora de entropía. Esta fuerza es la responsable del movimiento constante de los elementos del sistema. Un diseño con entropía busca que sus partes estén en constante movimiento y que el choque y encuentro de estos elementos detonen la creación de nuevos patrones de movimiento, subsistemas o elementos de encuentro.

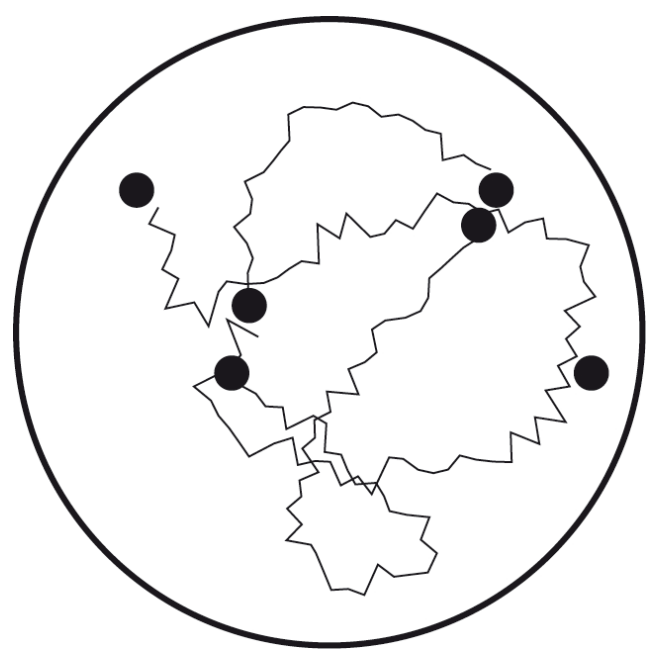

Fig. 1. Fuerza de entropía. Fuente: dibujo de los autores, 2016.

En la figura 2 se esquematiza a la fuerza de muerte. Esta potencia es la responsable de mantener al sistema en constante desarrollo, la función de la muerte en un sistema vivo es la de crear un intercambio energético-material. Lograr una degradación controlada de los componentes que asegure que el sistema se desarrolle o evolucione. Esta fuerza en el campo del diseño puede ser responsable de objetos con capacidad de transformación, desarrollo, mutación, intercambio de materiales, degradación controlada, reintegración al medio o de intercambios de energía con otros diseños o sistemas.

Si se observa la figura 3 se puede observar una esquematización de la fuerza de desarrollo en donde se comprende al sistema como un ente en movimiento direccional y cíclico. Esta fuerza ocasiona que el objeto se traslade en el tiempo y el espacio creando una forma con rastro y huella. Los sistemas vivos usan esta fuerza para crearse a sí mismos de forma constante y en cada momento son y están en una situación diferente lo que los direcciona hacia el crecimiento o el decrecimiento, se combina con las fuerzas de muerte y entropía para asegurarse que su desarrollo no sea estático, sino dinámico y con dirección. En el campo del diseño los objetos que logran incorporar la combinación de las tres fuerzas tienen una intención de cada una de ellas, su crecimiento o decrecimiento lleva una dirección establecida por el diseñador o es resultado de lo que se detona de alguna de las fuerzas anteriores. Un diseño con fuerza de desarrollo puede tener herramientas de prospectiva, planeación cronográfica. 
Una vez entendidas las tres fuerzas detonadoras de los sistemas vivos se pueden explicar sobre este esquema los comportamientos que definen a la autopoiesis. Estas características aseguran que se genere un patrón cíclico de antidispersión.

En la figura 4 se esquematiza el modelo completo. En donde la fuerza de dispersión se comporta como una tendencia a la disgregación de sus elementos componentes, responde a la fuerza de muerte y asegura que el sistema tenga potencia de movimiento. Este mismo comportamiento recluta a la tendencia de conservación, que esta esquematizada en la estructura contenedora, asegura una frontera definida que indica el momento en el que un sistema es y deja de ser. La tendencia de conservación asegura la resistencia constante a la dispersión y la degradación de los componentes y en contraposición con la fuerza de muerte genera la energía que necesita el objeto para subsistir. La tendencia relacional es el comportamiento asociativo de los componentes del sistema, pero en la suma de sus fuerzas también asegura el encuentro con otros sistemas vecinos o correlacionados. Esta tendencia de relación y encuentro asegura que el sistema trabaje en consonancia con el entorno en el que se encuentra, define la forma en que se alimenta, las vías de desalojo de desechos o residuos y posibilita la fusión, reproducción, desarrollo y metamorfosis del sistema.

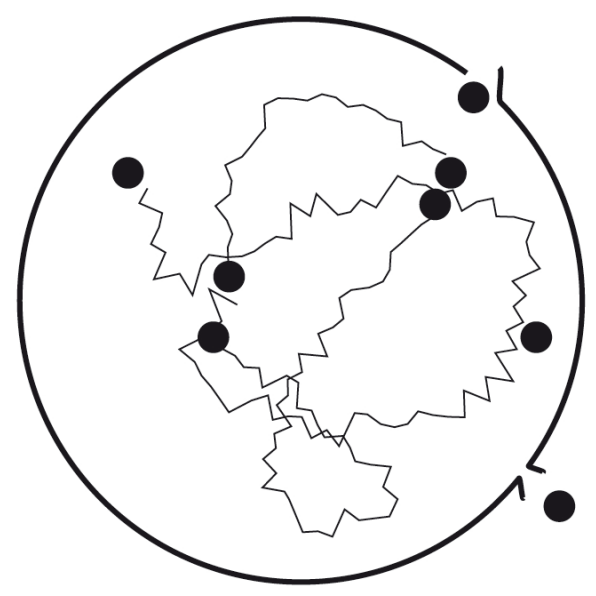

Fig. 2. Fuerza de muerte. Fuente: dibujo de los autores, 2016. 


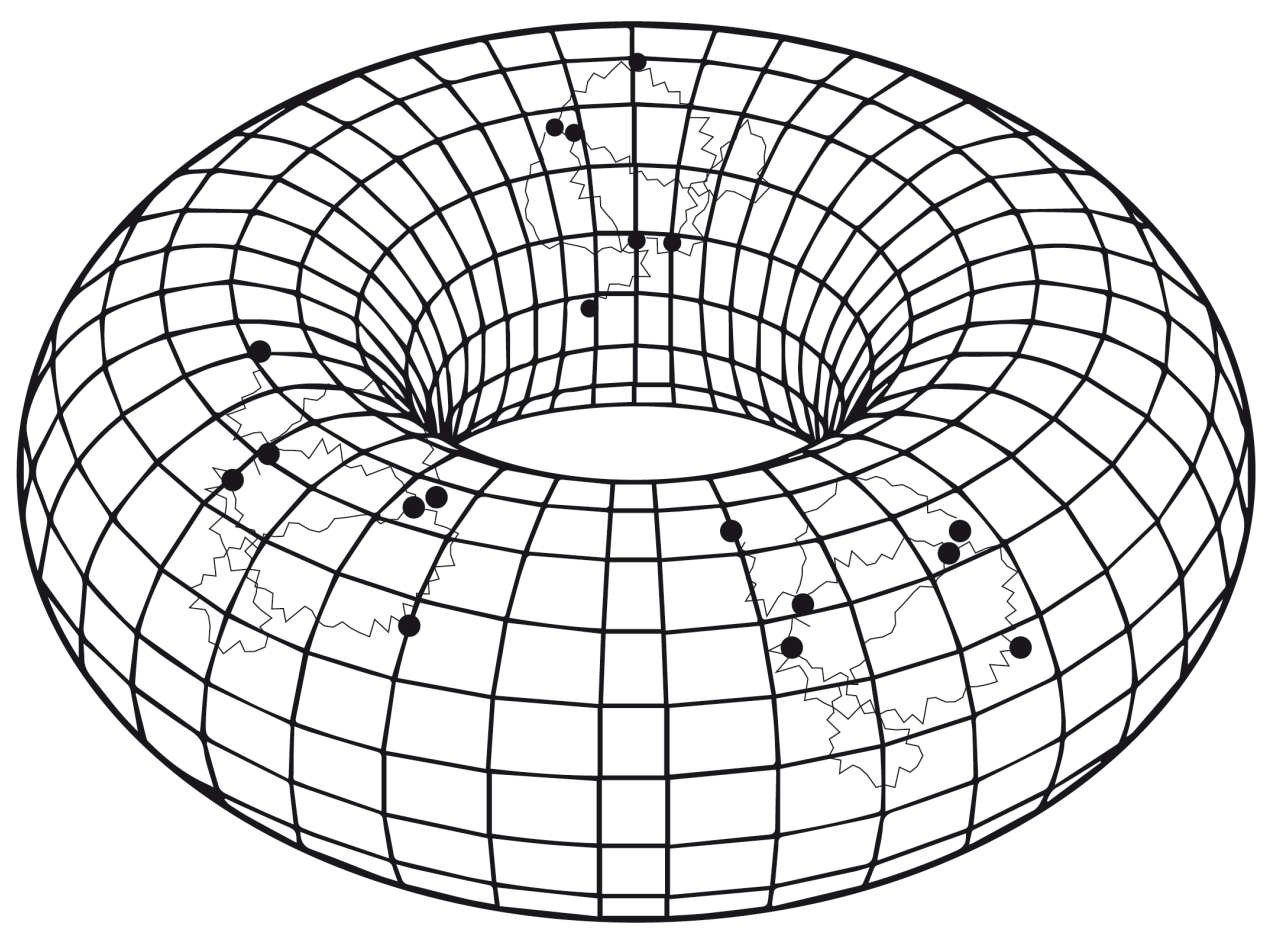

Fig. 3. Fuerza de desarrollo. Fuente: dibujo de los autores, 2016.

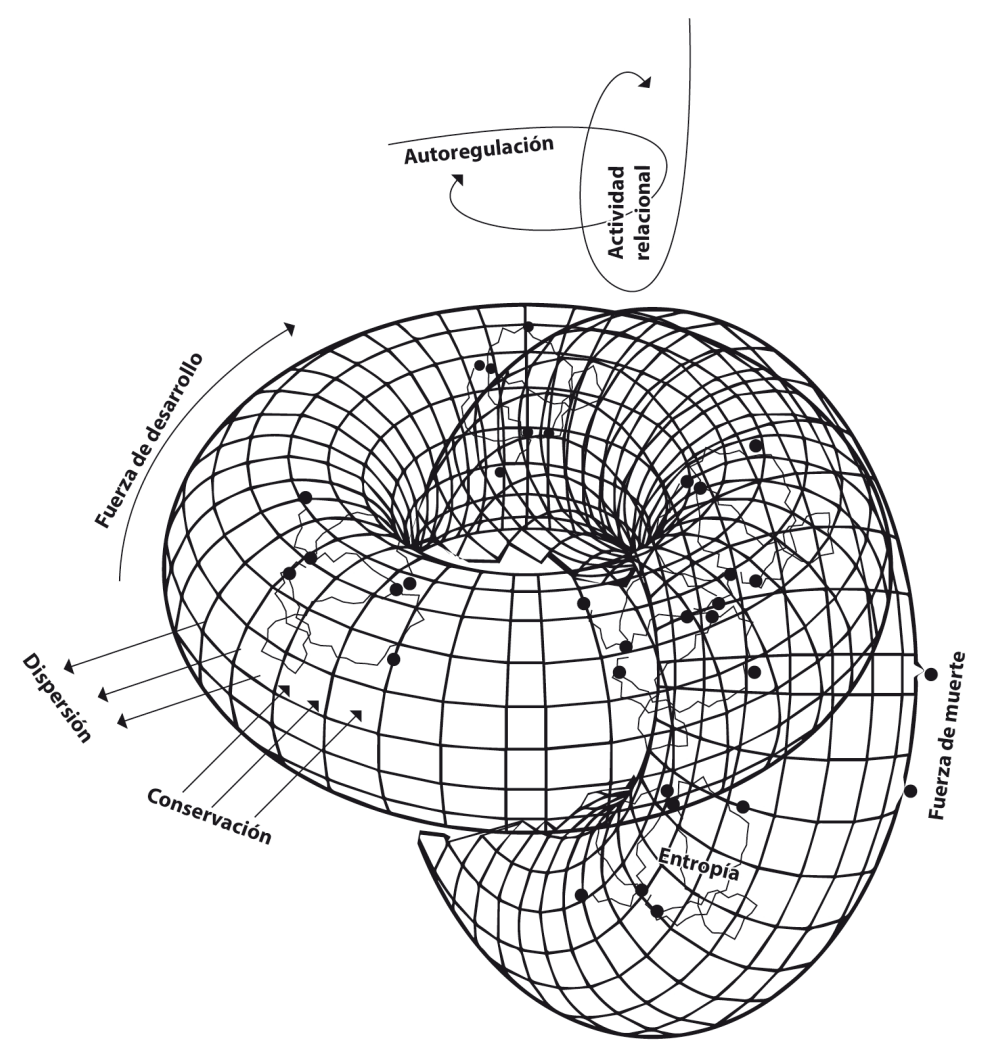

Fig. 4. Fuente: dibujo de los autores, 2016. 


\section{Aplicación al diseño}

La aplicación de este modelo autopoiético facilita la tarea de inspirarse en los modos y formas esenciales de la naturaleza para transportar las reflexiones resultantes a objetos concretos de diseño.

El modelo descrito proporciona una lista de atributos que en su contemplación constitutiva pueden integrarse al método de diseño que busca resultados biomiméticos.

Esta lista de atributos puede plantear una serie de índices paramétricos que pueden proporcionar escalas flexibles de aplicación.

La figura 5 se presenta una tabla de evaluación de objetos de diseño que busca intervenir de forma metodológica en el proceso de creación para enumerar y asignar un índice de valores que responde a preguntas específicas que a su vez corresponden a los aspectos esenciales del modelo autopoiético.

\begin{tabular}{|c|c|c|c|c|}
\hline $\begin{array}{l}\text { Fuerza o } \\
\text { característica }\end{array}$ & $\begin{array}{l}\text { Pregunta a la que busca } \\
\text { responder (aplicar cada } \\
\text { pregunta al objeto o } \\
\text { proceso evaluado) }\end{array}$ & $\begin{array}{l}\text { Nivel de respuesta en } \\
\text { escala del }-5 \text { al } 5 \text { (marcar } \\
\text { con una x el nivel en la } \\
\text { escala corresponde a la } \\
\text { respuesta que brinda el } \\
\text { objeto evaluado) }\end{array}$ & $\begin{array}{l}\text { Sumatoria parcial } \\
\text { (escribir con } \\
\text { número los puntos } \\
\text { obtenidos en cada } \\
\text { pregunta) }\end{array}$ & $\begin{array}{l}\text { Promedio de } \\
\text { puntuación } \\
\text { autopoiética (el } \\
\text { promedio parcial } \\
\text { de cada pregunta) }\end{array}$ \\
\hline Fuerza de muerte & $\begin{array}{l}\text { ¿Presenta una pérdida o } \\
\text { degradación de } \\
\text { componentes cuya acción } \\
\text { detona un proceso de } \\
\text { diferente? }\end{array}$ & $-5,-4,-3,-2,-1,0,1,2,3,4,5$ & & \\
\hline Fuerza entropía & $\begin{array}{l}\text { ¿Los componentes } \\
\text { presentan movimiento } \\
\text { dinámico interno? }\end{array}$ & $-5,-4,-3,-2,-1,0,1,2,3,4,5$ & & \\
\hline $\begin{array}{l}\text { Fuerza de } \\
\text { desarrollo }\end{array}$ & $\begin{array}{l}\text { ¿Presenta una evolución } \\
\text { transformadora a través } \\
\text { del tiempo o espacio? }\end{array}$ & $-5,-4,-3,-2,-1,0,1,2,3,4,5$ & & \\
\hline $\begin{array}{l}\text { Tendencia de } \\
\text { dispersión }\end{array}$ & $\begin{array}{l}\text { ¿Los componentes se } \\
\text { comportan de forma que } \\
\text { buscan su propia } \\
\text { reintegración al origen o } \\
\text { disgregación al entorno? }\end{array}$ & $-5,-4,-3,-2,-1,0,1,2,3,4,5$ & & \\
\hline $\begin{array}{l}\text { Tendencia de } \\
\text { conservación }\end{array}$ & $\begin{array}{l}\text { ¿Presenta una tendencia a } \\
\text { permanecer siendo lo que } \\
\text { es durante el ejercicio de } \\
\text { su función primordial? }\end{array}$ & $-5,-4,-3,-2,-1,0,1,2,3,4,5$ & & \\
\hline Autorregulación & $\begin{array}{l}\text { ¿Presenta capacidades } \\
\text { para detonar su propio } \\
\text { funcionamiento y proceso } \\
\text { sin intervención externa? }\end{array}$ & $-5,-4,-3,-2,-1,0,1,2,3,4,5$ & & \\
\hline \multirow[t]{2}{*}{$\begin{array}{l}\text { Actividad } \\
\text { relacional }\end{array}$} & $\begin{array}{l}\text { ¿tiene capacidad de } \\
\text { modulación, integración o } \\
\text { creación de su función o } \\
\text { nuevas funciones ante el } \\
\text { encuentro con el usuario } \\
\text { u otros objetos o } \\
\text { sistemas? }\end{array}$ & $-5,-4,-3,-2,-1,0,1,2,3,4,5$ & & \\
\hline & & & $\begin{array}{l}\text { Promedio total } \\
\text { (suma y promedia } \\
\text { todos los } \\
\text { resultados para } \\
\text { obtener el nivel } \\
\text { global de patrón } \\
\text { autópoiético en el } \\
\text { objeto evaluado) }\end{array}$ & \\
\hline
\end{tabular}

Fig. 5. Fuente: Hecho por los autores, 2016. 
La aplicación de esta evaluación tabulada pretende que las debilidades en las fuerzas o características puedan ser subsanadas mediante la aplicación estratégica de acciones pertinentes, con el objeto de fortalecer la concepción de un objeto de diseño que reproduzca la esencia de los sistemas vivos y aprovechar sus ventajas en sistemas hechos por el hombre.

Se sugiere la retroalimentación de la tabulación mediante la redacción de un texto que describa la estrategia de adecuación a seguir para cada sección en la que se requiera.

\section{Conclusiones}

La aplicación del modelo de análisis autopoiético brinda la oportunidad de realizar un trabajo de diseño biomimético de forma puntual y concreta.

Las características esenciales encontradas durante la realización del modelo presentado esquematiza de una forma asequible la dinámica de los componentes de la vida para ser entendidos como fuentes de información medible en los métodos de creación de todos los campos del diseño.

La existencia de otros modelos menos esquemáticos y adaptados para disciplinas distintas a la del diseño fortalece esta propuesta en su capacidad de aplicación en proyectos de distintos niveles y facetas disciplinares.

\section{REFERENCIAS}

LUHMAN, N. Organización y decisión, autopoiésis y entendimiento comunicativo. Anthropos, Barcelona 1977, España.

MATURANA, H. De máquinas y seres vivos. Autopoiésis: la organización de lo vivo. Editorial Universitaria/Universidad de Chile. Santiago de Chile 19985.

\section{Figuras:}

Fuente: dibujos de los autores, 2016. 